\title{
Synchronized TV Whitespace Spectrum Access
}

\author{
Yihenew Beyene, Kalle Ruttik and Riku Jäntti \\ Department of Communications and Networking \\ Aalto University, Finland \\ yihenew.beyene@aalto.fi, kalle.ruttik@aalto.fi, riku.jantti@aalto.fi
}

\begin{abstract}
Restrictions on adjacent channel interference leave large swath of spectrum unused. According to FCC, the adjacent channels of an active TV channel are excluded from unlicensed usage. This protection is crucial when estimating the amount of available TV whitespace. By its nature this limitation is technical. Strict primary user protection requirements render most of these adjacent channels unavailable for secondary transmissions. Better technical approaches would open up this reserve. In this paper we propose an OFDM based orthogonal transmission that matches DVB-T frame structure. The proposed scheme allows using immediate adjacent channels without the need of guard bands. We analyze how the proposed scheme generates interference to incumbent $\mathrm{TV}$ receivers and what constraints the proposed scheme has to meet. Simulation results show that the proposed scheme provides high signal-to-interference ratio even when the adjacent channel signal is relatively very strong. The scheme is an approach for relaxing the existing adjacent channel usage constraints.
\end{abstract}

\section{INTRODUCTION}

There have been extensive studies aiming at maximizing capacity of wireless systems operating in a limited radio resource. Today, there are advanced signal processing techniques [1], [2], [3] and network topologies [4] that enabled radio access technologies to have high spectral efficiency. However, the research effort has been mainly focused on maximizing capacity of a network that operates on a given block of frequency band.

Wireless communication networks suffer from signal distortions due to unpredictable channel variations and interference. Depending on the signal power level, adjacent channel interference (ACI) can severely degrade spectral efficiency of the network. ACI can be caused in two ways, by adjacent channel leakage and by poor adjacent channel selectivity (ACS). The leakage is caused by spillover of the neighboring channel transmitter signal. In OFDM signals, such interference comes from sidelobes. How well the receiver can reject the adjacent channel signal is mainly defined by receiver filters. Due to non-ideal filter, a wireless receiver could also suffer from ACI due to aliasing.

Unlike classical frequency division multiplexing (FDM), orthogonal frequency division multiplexing (OFDM) avoids the need for guard band when the messages for multiple receivers are sent over the same carrier. Latest radio access technologies like LTE, WiMAX, WLAN, DVB-T have adopted this modulation technique. Even though OFDM maximizes spectral efficiency of single carrier network, the traditional requirement for guard band between adjacent carriers is still in place. According to Federal Communications Commission (FCC), spectrum regulator for United States, unlicensed devices must operate outside the coverage contours of both co- and adjacent active TV channels [5]. In LTE systems the guard band takes nearly $10 \%$ of the total bandwidth [6]. The guard band is left for transition band of adjacent channel filter at the receiver that tries to reject interference on adjacent channels. In order to protect adjacent channels which might be used by different operators, a transmit power constraint is also applied.

Different techniques have been proposed to mitigate ACI. Transmitter side implementations include pre-equalization against distortions due to ACI and receiver's internal noise [7], and an extension of multi-antenna multiplexing scheme for adjacent channel signals [8]. The former approach requires feedback link and accurate estimate of the interference, and the latter requires full cooperation of all transmitters and receivers. Alternatively, receivers can do joint detection [9] or successive interference cancellation [10] where the receiver estimates and subtracts the adjacent channel signal before demodulating the desired signal.

In this paper we propose an open-loop joint transmitterreceiver pair design to obtain maximum performance. The proposed scheme requires no cooperation among transmitters and receivers. We extend OFDM properties for any two synchronized OFDM based signals transmitted on adjacent channels. Time and frequency synchronization of the two transmitters can be achieved by cyclic-prefix based blind autocorrelation which is sufficient to align OFDM symbols [11][13]. The proposed scheme allows networks with different radio access technologies to mitigate the interference they cause to each other without the need for guard band protection as long as they have equal symbol durations. To take full advantage of the scheme, all receivers need to oversample the signal sufficiently. However, it could be impractical to expect primary TV receivers to cancel interference from secondary users. On the other hand, secondary systems in TV whitespace can employ this technique to reduce strong interference from nearby TV signals. This enables reliable communication in TV whitespace with less transmit power while ensuring better protection for TV receivers. 

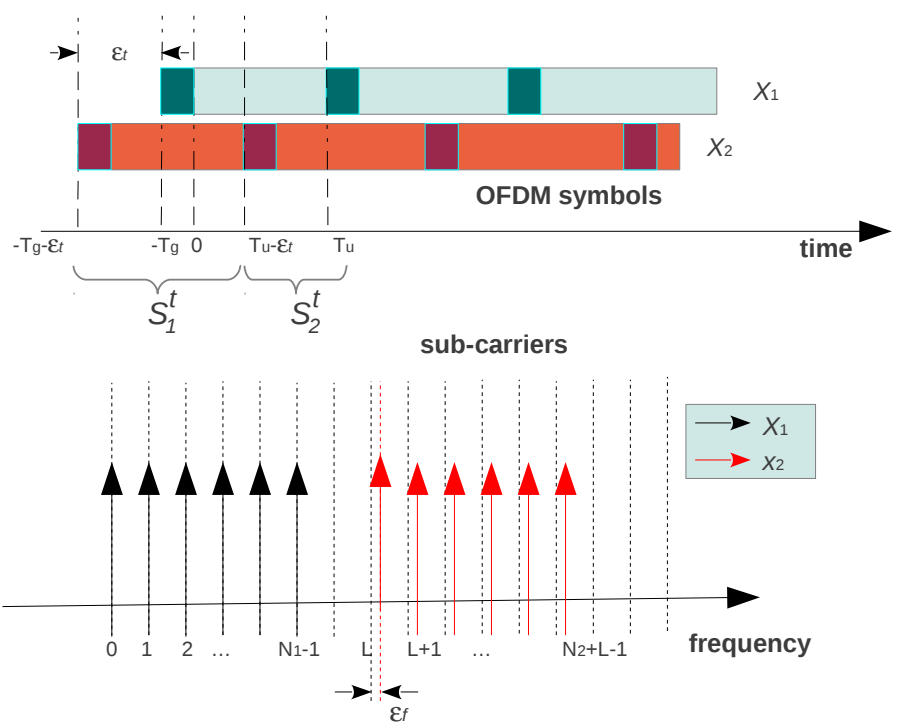

Fig. 1: Adjacent channel transmission

\section{SySTEM MODEL}

Consider two OFDM signals: primary channel (PCH) signal $x_{1}(t)$ with $N_{1}$ subcarriers: $\left\{0, \cdots, N_{1}-1\right\}$, and adjacent channel (ACH) signal, $x_{2}(t)$ having $N_{2}$ subcarriers: $\left\{L, \cdots, L+N_{2}-1\right\}$ where $L \geq N_{1}$. ACH transmitter tries to synchronize in time and frequency with the $\mathrm{PCH}$ transmission. Let $x_{2}(t)$ have carrier frequency offset (CFO) $\epsilon_{f}$, and timing offset (TO) $\epsilon_{t}$ relative to $x_{1}(t)$ as shown in Fig. 1. The CFO comes from non-ideal local oscillators. Both signals are assumed to have the same OFDM symbol duration.

$x_{1}(t)=\frac{1}{\sqrt{N_{1}}} \sum_{k=0}^{N_{1}-1} a_{k}^{(1)} e^{j 2 \pi k t / T_{u}}, \quad-T_{g} \leq t<T_{u}$
$x_{2}(t)=\left\{\begin{array}{l}\frac{\sqrt{\gamma}}{\sqrt{N_{2}}} e^{j 2 \pi\left(L / T_{u}+\epsilon_{f}\right) t} \sum_{k=0}^{N_{2}-1} b_{k}^{(1)} e^{j 2 \pi k\left(t+\epsilon_{t}\right) / T_{u}}, t \in S_{1}^{t} \\ \frac{\sqrt{\gamma}}{\sqrt{N_{2}}} e^{j 2 \pi\left(L / T_{u}+\epsilon_{f}\right) t} \sum_{k=0}^{N_{2}-1} b_{k}^{(2)} e^{j 2 \pi k\left(t+\epsilon_{t}-T_{g}-T_{u}\right) / T_{u}}, t \in S_{2}^{t}\end{array}\right.$

where $S_{1}^{t}=\left[-T_{g}-\epsilon_{t}, T_{u}-\epsilon_{t}\right), S_{2}^{t}=\left[T_{u}-\epsilon_{t}, 2 T_{u}+T_{g}-\epsilon_{t}\right)$. $T_{u}$ is the useful symbol duration, and $T_{g}$ is the guard period. $\gamma$ is normalized signal power of $x_{2}(t)$ with respect to unity power of $x_{1}(t)$. Received signal at the $\mathrm{PCH}$ receiver is given as

$$
y(t)=x_{1}(t)+x_{2}(t)+w(t), \quad-T_{g} \leq t<T_{u}
$$

where $w(t)$ is additive complex Gaussian noise. Let the sampling rate be $M / T_{u}, M \geq N_{1}$ where $M$ is the FFT window size. Define the normalized CFO as $\Delta_{f}=\epsilon_{f} T_{u}$ where $\left(1 / T_{u}\right)$ is the subcarrier interval. Assuming that $\epsilon_{t}=$ $\Delta_{n} T_{u} / M: \Delta_{n} \in \mathbb{Z}$, the discrete sampled signal is expressed as

$$
\begin{aligned}
y[n] & =y\left(n \frac{T_{u}}{M}\right)=x_{1}[n]+x_{2}[n]+w[n], n=0, \cdots, M-1 \\
& =\frac{1}{\sqrt{N_{1}}} \sum_{k=0}^{N_{1}-1} a_{k}^{(1)} e^{j 2 \pi n k / M}+w[n]+ \\
& +\left\{\begin{array}{l}
\frac{\sqrt{\gamma}}{\sqrt{N_{2}}} e^{j 2 \pi\left(L+\Delta_{f}\right) n / M} \sum_{k=0}^{N_{2}-1} b_{k}^{(1)} e^{j 2 \pi k\left(n+\Delta_{n}\right) / M}, n \in S_{1}^{n} \\
\frac{\sqrt{\gamma}}{\sqrt{N_{2}}} e^{j 2 \pi\left(L+\Delta_{f}\right) n / M} \sum_{k=0}^{N_{2}-1} b_{k}^{(2)} e^{j 2 \pi k\left(n+\Delta_{n}-G\right) / M}, n \in S_{2}^{n}
\end{array}\right.
\end{aligned}
$$

where $S_{1}^{n}=\left[0: M-\Delta_{n}-1\right], S_{2}^{n}=\left[M-\Delta_{n}: M-1\right]$. $\Delta_{n}$ is sample offset between $x_{2}(t)$ and $x_{1}(t) . a_{k}^{(l)}$ and $b_{k}^{(l)}$ are unity variance zero-mean complex modulated symbols mapped on $k^{t h}$ subcarrier of $l^{t h}$ OFDM symbol of $x_{1}(t)$ and $x_{2}(t)$ respectively.

After FFT operation, received signal on subcarrier $\tilde{k}$ is

$$
c_{\tilde{k}}=\sqrt{M / N_{1}} a_{\tilde{k}}^{(1)}+I_{\tilde{k}}+\nu_{\tilde{k}}, \quad \tilde{k} \in[0: M-1]
$$

where $\nu_{\tilde{k}}=\frac{1}{\sqrt{M}} \sum_{n=0}^{M-1} w[n] e^{-j 2 \pi \tilde{k} n / M}$ is the noise term, and $I_{\tilde{k}}$ is adjacent channel interference (ACI) from $x_{2}$.

$$
\begin{aligned}
& I_{\tilde{k}}=\sqrt{\gamma / N_{2} M} \sum_{k=0}^{N_{2}-1} e^{j 2 \pi k \Delta_{n} / M} \\
& \times\left[b_{k}^{(1)} \sum_{n=0}^{M-\Delta_{n}-1} e^{j 2 \pi\left(L+\Delta_{f}+k-\tilde{k}\right) n / M}+\right. \\
& \left.\quad b_{k}^{(2)} e^{-j 2 \pi k G / M} \sum_{n=M-\Delta_{n}}^{M-1} e^{j 2 \pi\left(L+\Delta_{f}+k-\tilde{k}\right) n / M}\right] .
\end{aligned}
$$

\section{AdJACENT CHANNEL INTERFERENCE}

The ACI term, $I_{\tilde{k}}$, comes from aliasing and imperfect time and frequency synchronization. When there is non-zero carrier offset $\Delta_{f} \in(-0.5,0.5]: \Delta_{f} \neq 0$ the ACI term reduces to

$$
\begin{aligned}
& I_{\tilde{k}}=\beta {\left[\sum_{k=0}^{N_{2}-1} b_{k}^{(1)} \frac{1-\alpha_{\tilde{k}}^{\left(M-\Delta_{n}\right)} e^{-j 2 \pi k \Delta_{n} / M}}{1-\alpha_{\tilde{k}} e^{j 2 \pi k / M}} e^{j 2 \pi k \Delta_{n} / M}+\right.} \\
&\left.\alpha_{\tilde{k}}^{M-\Delta_{n}} \sum_{k=0}^{N_{2}-1} b_{k}^{(2)} \frac{1-\left(\alpha_{\tilde{k}} e^{j 2 \pi k / M}\right)^{\Delta_{n}}}{1-\alpha_{\tilde{k}} e^{j 2 \pi k / M}} e^{-j 2 \pi k G / M}\right]
\end{aligned}
$$

with $\alpha_{\tilde{k}}=e^{j 2 \pi\left(L+\Delta_{f}-\tilde{k}\right) / M}, \beta=\sqrt{\gamma / N_{2} M}$ where $\left\{b_{i}^{(j)}\right\}$, are unity variance zero-mean independent random variables. Hence,

$$
\begin{array}{r}
E\left\{\left|I_{\tilde{k}}\right|^{2}\right\}=\beta^{2} \sum_{k=0}^{N_{2}-1} E\left\{\left|b_{k}^{(1)}\right|^{2}\right\}\left|\frac{1-\alpha_{\tilde{k}}^{\left(M-\Delta_{n}\right)} e^{-j 2 \pi k \Delta_{n} / M}}{1-\alpha_{\tilde{k}} e^{j 2 \pi k / M}}\right|^{2}+ \\
\beta^{2} \sum_{k=0}^{N_{2}-1} E\left\{\left|b_{k}^{(2)}\right|^{2}\right\}\left|\alpha_{\tilde{k}}^{M-\Delta_{n}}\right|^{2}\left|\frac{1-\left(\alpha_{\tilde{k}} e^{j 2 \pi k / M}\right)^{\Delta_{n}}}{1-\alpha_{\tilde{k}} e^{j 2 \pi k / M}}\right|^{2}
\end{array}
$$


with $E\left\{\left|b_{i}^{(j)}\right|^{2}\right\}=1,\left|\alpha_{\tilde{k}}^{M-\Delta_{n}}\right|^{2}=1$. This can be rewritten as

$$
\begin{gathered}
E\left\{\left|I_{\tilde{k}}\right|^{2}\right\}=\frac{\gamma}{N_{2} M} \sum_{k=0}^{N_{2}-1} \frac{2-\sqrt{2\left(1+\cos \phi_{1}\right)} \cos \left(\phi_{1} \Delta_{n}-\phi_{1} / 2\right)}{1-\cos \phi_{2}} \\
\text { where } \quad \phi_{1}=2 \pi \Delta_{f}, \quad \phi_{2}=\frac{2 \pi}{M}\left(L+\Delta_{f}+k-\tilde{k}\right) .
\end{gathered}
$$

When $\Delta_{f}=0$, we have

$$
\begin{array}{r}
E\left\{\left|I_{\tilde{k}}\right|^{2}\right\}=\frac{\gamma}{N_{2} M} \sum_{k=0}^{N_{2}-1}\left[\left|\sum_{n=0}^{M-\Delta_{n}-1} e^{j 2 \pi(L+k-\tilde{k}) n / M}\right|^{2}+\right. \\
\left.\left|\sum_{n=M-\Delta_{n}}^{M-1} e^{j 2 \pi(L+k-\tilde{k}) n / M}\right|^{2}\right] .
\end{array}
$$

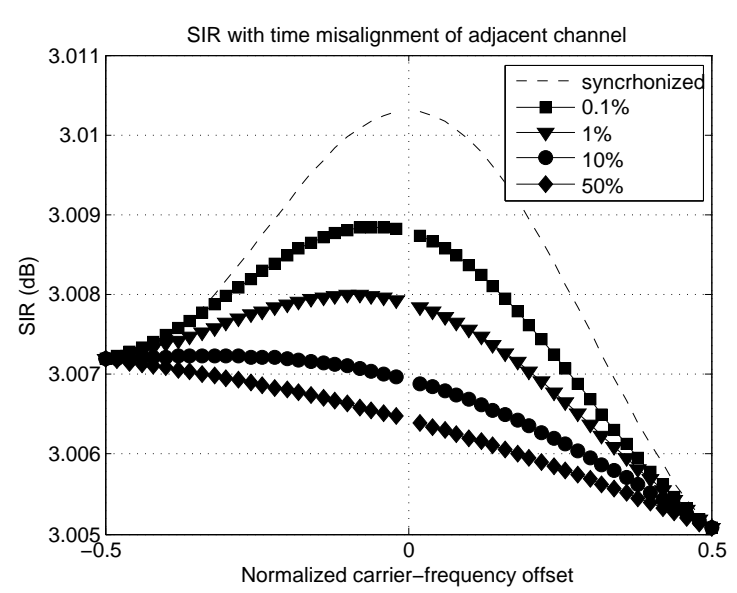

(a) $M=3072$

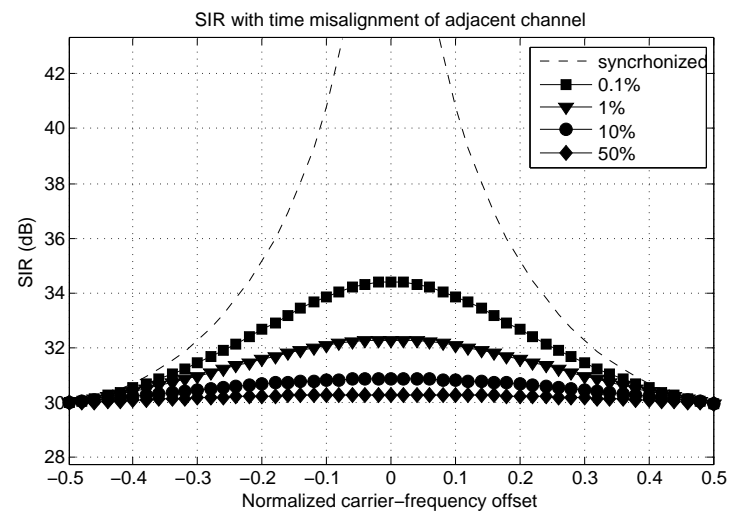

(b) $M=4096$

Fig. 2: Average SIR for different normalized sample offset values $\Delta_{n} / M . \gamma=0 d B, N_{1}=N_{2}=L=2048$.

Total interference power over the whole channel is the sum of interference terms given in (7). Average SIR over the whole bandwidth of the PCH signal has been given in (9). The interference comes from adjacent channel leakage (due to non-orthogonality) and aliasing when $M<L+N_{2}$.
Fig. 2 shows numerical results for SIR when different oversampling ratios, $M$, are used. It can be seen that when $M<L+N_{2}$, interference due to aliasing is dominant. Hence, time and frequency synchronicity add negligible gain on the SIR. On the other hand, when oversampling ratio is large enough such that spectrum folding does not happen, the received SIR can be improved significantly depending on how good the ACH transmitter can synchronize its transmission with the $\mathrm{PCH}$ transmitter in order to reduce the leakage power.

\section{A. Time synchronized}

The nice feature of OFDM is the possibility to extend the symbol duration cyclically for a given guard duration. Therefore, the receiver can start sampling any where within the guard interval without losing orthogonality of subcarriers.

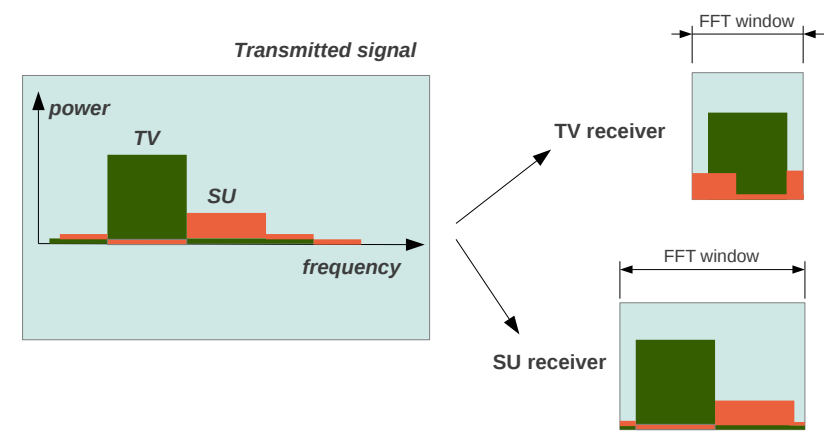

Fig. 3: Synchronized adjacent channel transmission.

Similarly, ACH signal can be synchronized in time with the $\mathrm{PCH}$ signal as long as the transmission starts during the guard period of PCH signal: $-G<\Delta_{n} \leq 0$. In this case the interference power has the same statistics (only the phase changes) as in perfect synchronization $\left(\Delta_{n}=0\right)$. Hence, we can assume that $\Delta_{n}=0$ for computing adjacent channel interference power. Average SIR in time-synchronized, $\Delta_{n}=0$, scenario can be derived as shown in (10).

\section{USING TV ADJACENT CHANNEL}

The TV band has been potential target for secondary access by unlicensed users using cognitive radios. This is due to the fact that the spectrum has not been used efficiently unlike cellular systems.

One way of using the TV band for secondary user (SU) communication is to overlay the secondary signal on top of the TV signal with very little power such that the TV receivers can tolerate the interference. In this case, the SU is required to relay the TV signal in order to compensate for the interference it generates. However, due to conservative SINR requirement to be met for all TV receivers, the secondary users spend most of their transmission power to relaying the TV signal rather than using it for communication. Moreover, TV signal interference cancellation at the secondary user suffers from channel estimation error due to pilot contamination [14]. Optimal schemes [15] also require cooperation between the 


$$
\begin{aligned}
& \operatorname{SIR}=\left\{\begin{array}{l}
\frac{N_{2} M^{2}}{\gamma}\left[\sum_{\tilde{k}=0}^{N_{1}-1} \sum_{k=0}^{N_{2}-1}\left|\sum_{n=0}^{M-\Delta_{n}-1} e^{j 2 \pi(L+k-\tilde{k}) n / M}\right|^{2}+\left|\sum_{n=M-\Delta_{n}}^{M-1} e^{j 2 \pi(L+k-\tilde{k}) n / M}\right|^{2}\right]^{-1}, \Delta_{f}=0 \\
\frac{N_{2} M^{2}}{\gamma}\left[\sum_{\tilde{k}=0}^{N_{1}-1} \sum_{k=0}^{N_{2}-1} \frac{2-\sqrt{2\left(1+\cos \left(2 \pi \Delta_{f}\right)\right.} \cos \left(2 \pi\left[\left(L+\Delta_{f}+k-\tilde{k}\right) \Delta_{n} / M-\Delta_{f} / 2\right]\right)}{1-\cos \left(2 \pi\left(L+\Delta_{f}+k-\tilde{k}\right) / M\right)}\right]^{-1}, \Delta_{f} \in(-0.5,0.5]: \Delta_{f} \neq 0
\end{array}\right. \\
& \operatorname{SIR}_{\text {sync }}=\frac{N_{2} M^{2}}{\gamma}\left[\sum_{\tilde{k}=0}^{N_{1}-1} \sum_{k=0}^{N_{2}-1} \frac{1-\cos \left(2 \pi\left(L+\Delta_{f}+k-\tilde{k}\right)\right)}{1-\cos \left(2 \pi\left(L+\Delta_{f}+k-\tilde{k}\right) / M\right)}\right]^{-1}
\end{aligned}
$$

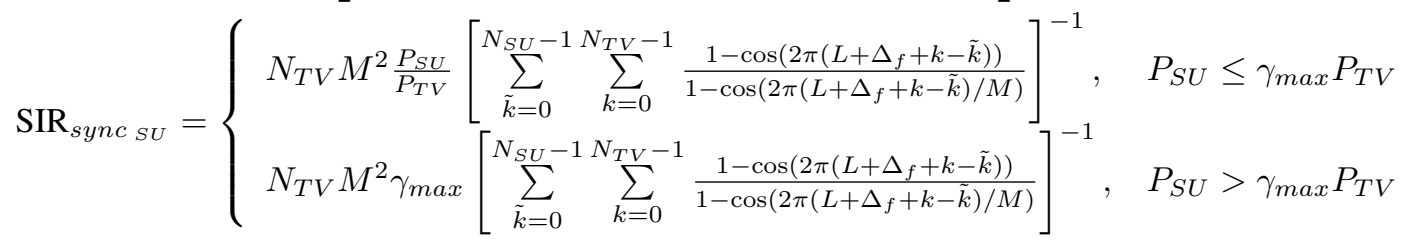

$$
\begin{aligned}
& \operatorname{SIR}_{\text {sync SU }}^{(A C R)}= \begin{cases}N_{T V} M^{2} \frac{P_{S U}}{P_{T V}}\left[\sum_{\tilde{k}=0}^{N_{S U}-1}\left|H\left[\tilde{k}-L-N_{T V} / 2\right]\right|^{2} \sum_{k=0}^{N_{T V}-1} \frac{1-\cos \left(2 \pi\left(L+\Delta_{f}+k-\tilde{k}\right)\right)}{1-\cos \left(2 \pi\left(L+\Delta_{f}+k-\tilde{k}\right) / M\right)}\right]^{-1}, & P_{S U} \leq \gamma_{\max } P_{T V} \\
N_{T V} M^{2} \gamma_{\max }\left[\sum_{\tilde{k}=0}^{N_{S U}-1}\left|H\left[\tilde{k}-L-N_{T V} / 2\right]\right|^{2} \sum_{k=0}^{N_{T V}-1} \frac{1-\cos \left(2 \pi\left(L+\Delta_{f}+k-\tilde{k}\right)\right)}{1-\cos \left(2 \pi\left(L+\Delta_{f}+k-\tilde{k}\right) / M\right)}\right]^{-1}, & P_{S U}>\gamma_{\max } P_{T V}\end{cases}
\end{aligned}
$$

two transmitters.

Another alternative is to access temporally and/or spatially unused TV bands, whitespaces. Ideally, the SU can have interference free communication by interleaving its transmission on available TV whitespaces. When whitespaces on immediate adjacent channel are used, leakage power from TV signal, however, could severely affect SU communication and canceling such interference is not straightforward problem.

An efficient interleaving of SU signal on TV adjacent channel that mitigates the interference can be achieved through synchronization of the two transmissions. SU receiver oversamples the signal sufficiently enough such that the TV signal spectrum does not fold onto SU signal. Hence the TV interference on the SU signal comes only from leakage due to frequency error as illustrated in Fig. 3.

Target signal-to-interference plus noise ratio (SINR) for the TV receiver, $\operatorname{SINR}_{P C H \text { min }}$ puts a limit on the maximum power that can be used for SU signal. In non-cooperative scenario where the TV receiver may not oversample the signal sufficiently, the SU may totally overlap on the TV signal due to aliasing, and synchronicity has negligible impact on the received SINR. From the TV receiver point of view, such adjacent channel interference is similar to overlay type transmission except that the adjacent channel signal is attenuated by the channel filter before it is sampled.

$\mathrm{SINR}_{T V, \text { min }}$ can be mapped into ratio between received
TV signal power $P_{T V}$ and SU signal power $P_{S U}$ given by $\gamma=P_{S U} / P_{T V} \leq \gamma_{\max }$. To meet this constraint, the SU may allocate fraction of its power, $\alpha P_{S U}$, for relaying the TV signal depending on available power budget for SU transmitter. Fig. 4 shows the relaying fraction for a given SU power budget and received TV signal power.

$$
\alpha= \begin{cases}0 & P_{S U} \leq \gamma_{\max } P_{T V} \\ \frac{P_{S U}-\gamma_{\max } P_{T V}}{\left(1+\gamma_{\max }\right) P_{S U}} & P_{S U}>\gamma_{\max } P_{T V}\end{cases}
$$

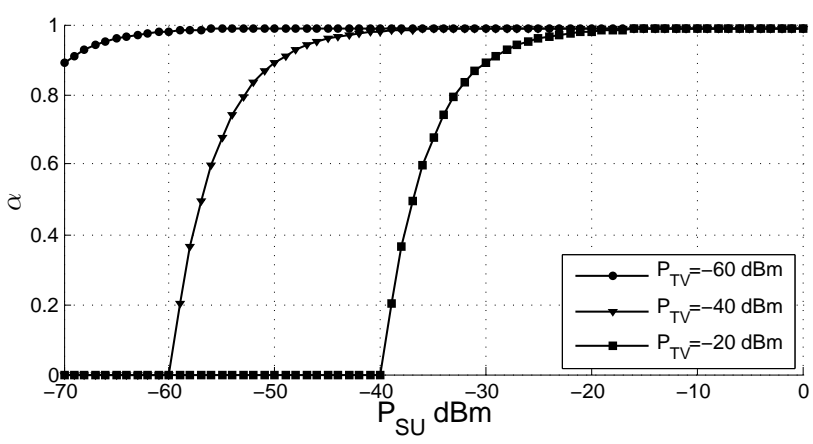

Fig. 4: TV signal relaying fraction when $\gamma_{\max }=-20 d B$.

When SU and TV transmissions are time-synchronized (transmissions start within the guard period), we can assume $\Delta_{n}=0$. Hence, SIR at SU receiver is given in (11). Fig. 5 shows SIR of secondary user in DVB-T band. In practice, wireless transmitters have adjacent channel rejection (ACR) filters that suppress sidelobes. For examples, the 

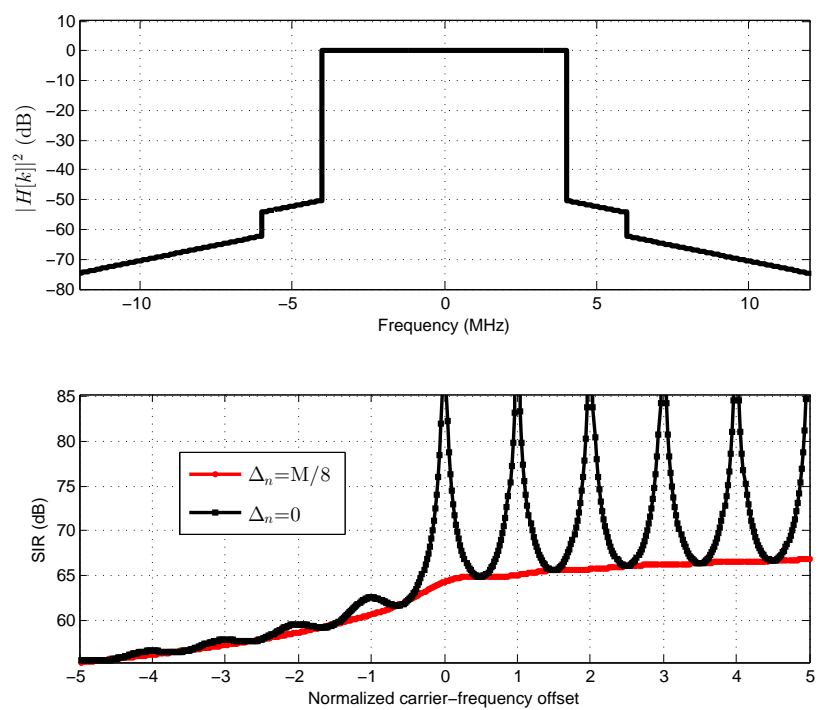

Fig. 6: DVB-T spectral mask (top) and received SIR $_{S U}$ (bottom) in adjacent channel: $\gamma_{\max }=-20 \mathrm{~dB}, N_{T V}=N_{S U}=$ $L=1705$ ( $2 \mathrm{~K}$ mode), $M=4096$.

ETSI (European Telecommunications Standards Institute) recommendation [16] guarantees that out-of-band emissions of DVB-T transmitters shall be attenuated by at least $40 \mathrm{~dB}$.
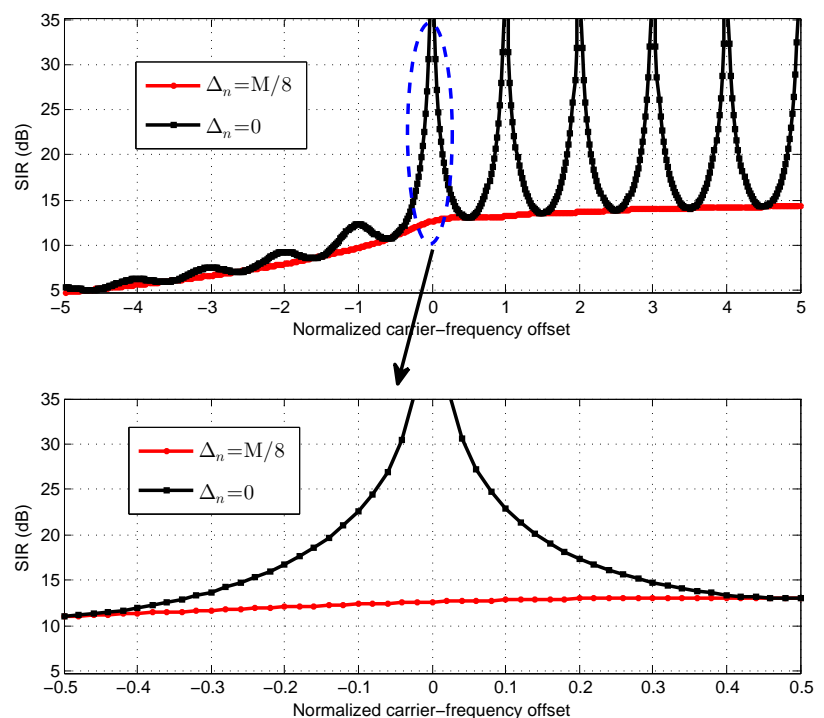

Fig. 5: SIR $_{S U}$ in DVB-T adjacent channel: $\gamma_{\max }=-20 \mathrm{~dB}$, $N_{T V}=N_{S U}=L=1705$ ( $2 \mathrm{~K}$ mode), $M=4096$.

Consider aliasing free secondary receiver, $M>L+N_{T V}$. The interference comes only from out-of-band leakage of the TV signal. This interference is attenuated by the spectral mask as defined in [16]. Hence, taking ACR filter at the TV transmitter into consideration, time-synchronized SIR would be as given in (12) where $H[k]$ is frequency response of lowpass ACR filter applied to baseband TV signal. Fig. 6 plots received SIR in the presence of spectral mask.

\section{CONCLUSiON}

In this work, the benefit of synchronized adjacent channel transmission has been presented. We analyze how two OFDM based adjacent channel signals can coexist without causing excessive interference to one another. The proposed scheme orthogonalized the secondary signal and TV signal. It allows secondary users to reliably communicate in presence of strong TV signal in adjacent channel. Since all DVB receivers use oversampling we also observe protection of incumbent TV receivers. Future work should take into account the impact inter-modulation and distortions due to non-linear amplifiers.

\section{REFERENCES}

[1] R. Irmer, H. Droste, P. Marsch, M. Grieger, G. Fettweis, S. Brueck, H. P. Mayer, L. Thiele, and V. Jungnickel, "Coordinated multipoint: Concepts, performance, and field trial results," Communications Magazine, IEEE, vol. 49, no. 2, pp. 102-111, 2011.

[2] M. Sawahashi, Y. Kishiyama, A. Morimoto, D. Nishikawa, and M. Tanno, "Coordinated multipoint transmission/reception techniques for LTE-advanced [Coordinated and Distributed MIMO]," Wireless Communications, IEEE, vol. 17, no. 3, pp. 26-34, 2010.

[3] A. Paulraj, D. Gore, R. Nabar, and H. Bolcskei, "An overview of MIMO communications - a key to gigabit wireless," Proceedings of the IEEE, vol. 92, no. 2, pp. 198-218, 2004

[4] A. Khandekar, N. Bhushan, J. Tingfang, and V. Vanghi, "LTE-advanced: Heterogeneous networks," in Wireless Conference (EW), 2010 European, 2010, pp. 978-982.

[5] FCC-10-174, "In the Matter of Unlicensed Operation in the TV Broadcast Bands: Second memorandum opinion and order," 2010.

[6] “3GPP TR 25.814 v7.1.0 - $3^{r d}$ Generation Partnership Project; Technical Specification Group Radio Access Network; Physical layer aspects for evolved Universal Terrestrial Radio Access (UTRA) (Release 7)," 2006.

[7] G. Foudazi and H. Shafiee, "Adjacent channel rejection in OFDM wireless local area networks," in Wireless and Optical Communications Networks, 2006 IFIP International Conference on, 2006, pp. 4 pp.-4.

[8] D. Gidony and I. Kalet, "Adjacent channel interference cancellation for MSK-type signals using antenna diversity in rayleigh fading environment," Communications, IEEE Transactions on, vol. 52, no. 2, pp. 317325, 2004.

[9] H. Arslan, S. C. Gupta, G. Bottomley, and S. Chennakeshu, "New approaches to adjacent channel interference suppression in FDMA/TDMA mobile radio systems," Vehicular Technology, IEEE Transactions on, vol. 49, no. 4, pp. 1126-1139, 2000.

[10] H. Osada, H. Nishimura, M. Inamori, and Y. Sanada, "Adjacent Channel Interference Cancelation in Fractional Sampling OFDM Receiver," in Vehicular Technology Conference (VTC Fall), 2011 IEEE, 2011, pp. 15.

[11] J.-J. van de Beek, M. Sandell, and P. Borjesson, "ML estimation of time and frequency offset in OFDM systems," Signal Processing, IEEE Transactions on, vol. 45, no. 7, pp. 1800-1805, 1997.

[12] S. Ma, X. Pan, G.-H. Yang, and T.-S. Ng, "Blind Symbol Synchronization Based on Cyclic Prefix for OFDM Systems," Vehicular Technology, IEEE Transactions on, vol. 58, no. 4, pp. 1746-1751, 2009.

[13] B. Su and P. Vaidyanathan, "Blind block synchronization algorithms in cyclic prefix systems," in Circuits and Systems, 2008. ISCAS 2008. IEEE International Symposium on, 2008, pp. 137-140.

[14] Y. Beyene, K. Ruttik, and R. Jantti, "Effect of secondary transmission on primary pilot carriers in overlay cognitive radios," in Cognitive Radio Oriented Wireless Networks (CROWNCOM), 2013 8th International Conference on, 2013, pp. 111-116.

[15] M. Costa, "Writing on dirty paper (corresp.)," Information Theory, IEEE Transactions on, vol. 29, no. 3, pp. 439 - 441, may 1983.

[16] "ETSI EN 302296 v1.1.1 - Candidate Harmonized European Standard (Telecommunications series); Electromagnetic compatibility and Radio spectrum Matters (ERM); Transmitting equipment for the digital television broadcast service, Terrestrial (DVB-T)," Tech. Rep., 2005. 